\title{
La Andragogía como disciplina propulsora de conocimiento en la educación superior
}

\section{Andragogy as a Knowledge Driving Discipline in Higher Education}

\author{
Paula Alonso Chacón ${ }^{1}$ \\ Centro de Estudios Generales \\ Universidad Nacional \\ Heredia, Costa Rica \\ alonsopaula24@hotmail.com
}

Recibido 23 de enero de 2012 • Aceptado 23 de febrero 2012

\begin{abstract}
Resumen. Este artículo informa sobre la diacronía (panorama histórico) de la Andragogía y de su incursión como disciplina en el contexto de la educación universitaria. Asimismo, a partir de los principios andragógicos, del proceso de pensamiento del adulto y de la experiencia laboral, se propone al acto andragógico como una opción educativa por implementarse en la educación superior costarricense, para desarrollar competencias cognitivas y metacognitivas en los estudiantes universitarios, en forma simultánea, con las distintas áreas académicas, mediante la reproducción de la mayéutica socrática, que se estructura en el ciclo de aprendizaje experiencial propuesto por Kolb.
\end{abstract}

Palabras claves. Andragogía, educación superior, adulto, competencia cognitiva, competencia metacognitiva, docente universitario.

Abstract. This paper exposes the diachronic (historical overview) of Andragogy (or Adult Education) and its introduction as a discipline in the context of university education. Based on the andragogical principles of the adult thought process and the work experience, this study sets out Adult Education as an education option to be implemented in

higher education, in Costa Rica, to develop cognitive and meta-cognitive competencies in the university students, in the different academic areas simultaneously, by reproducing the Socratic maieutics, which is structured within the Kolb's experiential learning cycle.

Keywords. Andragogy, higher education, adult, cognitive competence, meta-cognitive competence, university professor.

\footnotetext{
Estudiante de la Maestría en Educación con énfasis en Docencia Universitaria en la Universidad Nacional. Licenciada en Literatura y Lingüística con énfasis en Español como Segunda Lengua por la Universidad Nacional. Bachiller en Filología Española por la Universidad de Costa Rica. Ha sido profesora de Español como segunda lengua en Cuerpo de Paz, Costa Rica; así como en otras instituciones privadas y profesora de Español en el Colegio Yurusti. Se ha capacitado para evaluar la competencia comunicativa de estudiantes de Español como Segunda Lengua. Ha estudiado francés por varios años y ha obtenido el certificado internacional DELF emitido por el gobierno francés. Actualmente, se desempeña como docente en el Centro de Estudios Generales de la Universidad Nacional en el área de Filosofía y Letras.
} 


\section{Introducción}

Actualmente, gran parte de la docencia universitaria tiene como fin último la transmisión del conocimiento, por cuanto el docente, visto como un ser acabado en su proceso de aprendizaje, se reviste de autoridad para dictar materia y el discente se limita a recibir pasivamente y, sin mucho cuestionamiento, la información dada. Esta relación no solo irrespeta la condición de adulto del estudiante, sino que también mengua el desarrollo de competencias cognitivas y metacognitivas, deseables de estimular en cualquier ser humano. En consecuencia, resulta indispensable que el docente se informe sobre la Andragogía como disciplina educativa y visualice al acto andragógico como una herramienta idónea, para horizontalizar la relación citada arriba e impulsar una formación integral en el estudiante.

Este artículo tiene como objetivo brindar un panorama sobre la Andragogía como disciplina educativa, lo cual coadyuvará a establecer la importancia de su implementación en las aulas universitarias. Se espera que el docente universitario se familiarice con los principales lineamientos del acto andragógico y sus beneficios, a fin de que los integre, paulatinamente, en su quehacer académico.

\section{Antecedentes de la andragogía}

Desde la vida intrauterina y tras el alumbramiento, el homo sapiens se perfila como un ser dependiente de otros; por una parte, para sobrevivir, ya que su base psicobiológica le imposibilita su autonomía y; por otra parte, está destinado a vivir en sociedad, lo que implica relacionarse con sus homólogos constantemente, con el fin de construirse y de descubrirse a sí mismo.

En este sentido, el ser humano como ser inacabado, pero poseedor de gran plasticidad cerebral $^{2}$, debe formar su personalidad y adaptarse a los cambios sociales, en virtud de alcanzar el perfeccionamiento a través de toda su vida, para evolucionar. A esta capacidad humana se le denomina educabilidad (Tarrio, s. f.).

Además, el hombre es educable porque está dotado de pensamiento abstracto, lo cual lo habilita para utilizar competencias cognitivas como la conciencia del yo y del otro, los conceptos de espacio, tiempo y la capacidad de cambiar el pensamiento para ejecutar acciones más complejas (Rivera, 1998). También, emplea competencias metacognitivas como la toma de decisiones fundamentada, hecho que lo aleja del actuar instintivo. Así, el liberarse de conductas instintivas le permitirá educarse mediante la aprehensión de los estímulos del medio ambiente y el enriquecimiento de las vivencias que lo transformarán como ser individual y como miembro de un grupo (Tarrio, s. f.).

A la luz de la perspectiva anterior, en Occidente, surge, entre los pensadores griegos, una inquietud evidente por la educabilidad de los ciudadanos. El primero de ellos es Platón (427-347 a. C), quien esboza el concepto de educación en términos de la dualidad humana alma-cuerpo. De este modo, el hombre debe liberarse de la esclavitud del cuerpo que mantiene al alma confinada, puesto que la esencia de este es el alma y el alma es, predominantemente, razón. ¿Y cómo se libera? mediante la verdadera educación. Para Platón, el hombre educado es el que se esfuerza

2 Por plasticidad cerebral se entiende: “(...) la adaptación funcional del sistema nervioso central (SNC) para minimizar los efectos de las alteraciones estructurales o fisiológicas sea cual fuere la causa originaria (...). (Pascual-CastroViejo, 1996, p. 1361) 
por cuestionar y desentrañar el significado de las cosas, con el objeto de superar su banalidad. La verdadera educación implica el adoptar una nueva visión de mundo (Banchio, 2004), que se explica a través de la siguiente cita:

El hombre educado es aquél [sic] que, comprendiendo que el mundo sensible es sólo [sic] una imagen confusa del realmente real, se eleva de lo sensible, mudable y material a lo eterno, inmutable, inteligible e inmaterial, a las ideas; y al hacerlo descubre que está llamado a vivir como un dios, en trato directo con lo Eterno. (El dualismo antropológico de Platón, párr. 5)

La idea anterior se origina del Mito de la caverna, que constituye un pasaje de La república, en el cual se determina el estado de la naturaleza humana según esté permeada o no por la educación. Así pues, la historia relata que un grupo de hombres vivía dentro de una caverna, que los separaba del mundo exterior mediante un camino escabroso. Estos, naturalmente, pensaban que las sombras de ese otro mundo que se reflejaba en la caverna era el único existente, viviendo engañados por la ignorancia. Un día, uno de los hombres logra ver el mundo exterior y se enceguece por la luz que nunca había visto y, cuando logra tener un panorama claro y completo, se da cuenta de que la caverna era un oscuro submundo.

Como muestra de solidaridad, este hombre vuelve a la caverna, para contarles a los demás que él ha visto la luz, la verdadera realidad (Banchio, 2004). En consecuencia, la enseñanza que encierra esta alegoría responde al proceso que pasa todo educando desde la oscuridad o la ignorancia hasta llegar a concientizarse de la luz o de la sabiduría que lo transportará a un mundo sublime.

Como complemento al Mito de la caverna, Platón también se refiere al Mito del carro alado en el libro Fedro, para destacar las características del alma, que van en detrimento de la razón $\mathrm{y}$, por ende, inducen un comportamiento impulsivo. Este relato compara el alma humana con un carro alado y tirado por dos caballos, que es manipulado por un auriga. Ambos caballos son de diferente naturaleza, lo que hace difícil manejarlos. El auriga representa a la razón y los caballos a las partes concuspicible e irascible del alma. El viaje del carro alado al cielo se torna afanoso, porque el caballo de la concupiscencia no permite que el carro se eleve. De ahí que el hombre no debe alimentar las pasiones, sino la virtud de la razón y del autocontrol sobre sus acciones.

El segundo pensador que aporta a la Educación es Sócrates (469-399 a.C.), mediante la mayéutica, método filosófico de investigación y de enseñanza. Este consiste en entablar un diálogo entre el maestro y el discípulo estructurado en cuatro estadios: en el primero se plantea una pregunta del tipo: ¿qué es?; en el segundo, el discípulo contesta la pregunta, que es rebatida por el maestro, lo que produce confusión y malestar en el discípulo; en el tercero, se suscita una discusión sobre el tema. Aquí, el objetivo del método radica en estimular definiciones más generales y precisas, y en el cuarto continúa la discusión hasta que el discípulo alcanza el conocimiento general, exacto y riguroso de dicho tema.

Valga señalar que la fundamentación del método socrático parte de la siguiente premisa: el maestro no deposita información en el discípulo sobre un tema particular, sino que es este último, quien extrae de sí mismo el conocimiento que está oculto en su psique, por medio del análisis crítico, generado por la oportuna conducción de un mediador (Fingermann, 2011).

El tercer filósofo que contribuye al acto educativo es Aristóteles (384-322 a. C), quien sostiene que el fin de la existencia es la conquista de la felicidad. Para alcanzarla, el hombre debe cultivar las 
relaciones sociales y el espíritu. Por ello, sobresalen en este estudioso la educación del carácter, la cual es innata, pues el hombre es un ser social; es decir, que solamente por medio de la interacción con otros individuos el hombre se hace hombre, que significa ser un buen hombre.

De ahí que unos se expliquen a otros cómo debe ser su conducta. Ahora bien, esta educación debe ejercitarse a través de toda vida, puesto que el hombre es dependiente e imperfecto. En consecuencia, la educación se entiende como un proceso de perfeccionamiento y, por tanto, abarcará todo el ciclo vital.

Asimismo, Aristóteles cree en el carácter funcional de la educación; sin duda, se debe practicar aquello que se desea aprender. En este proceso, el educador constituye un guía que propone fines al quehacer educativo y que acompaña al educando en el fortalecimiento de las buenas inclinaciones (Hernández, s. f.).

Sin embargo, no solo los griegos se manifiestan en cuanto al acto educativo del hombre. Como resultado de las multitudes desocupadas y desorientadas a causa de la Revolución Francesa, Nicolas Condorcet, en 1793, publica un proyecto de decreto sobre la instrucción pública llamado Cinco memorias sobre la instrucción pública, en donde expresa que la República debe velar por la independencia de tal instrucción para masificarla.

En su planteamiento esboza un nuevo concepto de educación, que se vislumbra como educación permanente mediada por la acción y la participación (Álvarez, 1977):

Ofrecer a todos los individuos de la especie humana los medios de satisfacer sus necesidades de lograr un bienestar, de conocer y ejercer su derecho, de comprender y llenar sus deberes, de asegurar de cada uno de ellos las facilidades de perfeccionar su industria, de hacerse capaz de ejercer funciones sociales para las que tiene el derecho de ser llamado, de desarrollar toda la amplitud de las aptitudes que ha recibido de la naturaleza y establecer así entre las ciudades una igualdad de hechos convirtiendo en realidad la igualdad política reconocida por la ley, lo cual ha de ser el primer objetivo de una instrucción racional. (Álvarez, 1977, pp. 1-2)

Hasta aquí, se ha hecho alusión a ideas que, de forma patente, conforman el insumo para los futuros principios andragógicos. Estas ideas son: la liberación y el perfeccionamiento del ser humano (educación permanente) mediante el conocimiento adquirido por medio del acto educativo, y la elaboración del conocimiento por parte del mismo hombre, entendido como un descubrimiento a partir de la reflexión. Pero, no es sino hasta 1833 cuando el maestro de primaria alemán Alexander Kapp retoma el arquetipo platónico de educación y acuña el término Andragogía, derivado de la etimología griega $\alpha v \delta \varrho o s$ (hombre) y ó $\gamma \omega$ (conduzco). En consecuencia, esta se entiende como el arte y la ciencia que facilita el proceso de aprendizaje de los adultos (Instituto Nacional para la Educación de Adultos [INEA], 2007).

El vocablo Andragogía surge en oposición al término Pedagogía ( $\pi \alpha \iota \delta ı v v$, niño y á $\gamma \omega$, conduzco), pues Kapp considera a esta última como una disciplina que restringe el proceso educativo a la niñez, sin extenderlo a la vida total del ser humano y que, además, se centra más en la transmisión de conocimientos que en su construcción.

De hecho, él plantea, empíricamente, su inaplicabilidad en la educación de adultos, ya que los postulados pedagógicos no colman las demandas del adulto como un ser capaz y con un cúmulo de experiencias que rigen, en gran parte, el contexto educativo. 
Sin embargo, su idea es rechazada por la interpretación de Johann Herbart, quien es fiel creyente de la instrucción como acto educativo, que moldea el deseo y la voluntad de las personas, lo cual sume al término Andragogía en el olvido por casi un siglo (Sánchez, 1989; Mijangos, s. f.).

Luego, en 1921, el sociólogo alemán Eugen Rosenstock reanuda el término Andragogía y asevera que la Pedagogía es inaplicable a la educación de adultos, la cual requiere una base filosófica, unos métodos, un currículo y una relación entre profesor-alumno distintas de lo concebido en la educación para niños. También, hace hincapié en que la educación de adultos no debe circunscribirse dentro de un aula, sino que el proceso andragógico tiene una relación directa con la vida y con la formación profesional de los adultos, a fin de facilitarles la adquisición cognitiva y las destrezas indispensables para ello.

Pese a su gran aporte, estas ideas no gozan de aceptación ni en Europa ni en Estados Unidos hasta que ambos contextos experimentan las secuelas de la Segunda Guerra Mundial, cuando se enfrentan a una masa de ciudadanos discapacitados y analfabetos, a quienes es preciso reeducar, para que se incorporen a la fuerza laboral (Álvarez,1977; Lépiz, 2011).

\section{La Andragogía en el contexto de la educación superior}

En relación con lo expuesto en líneas anteriores, la reciente teoría educativa denominada Andragogía se consolida mediante la definición del adulto, la psicología de su aprendizaje, cuatro postulados y siete elementos, que caracterizan al acto andragógico. Dichos elementos se describen a continuación, respectivamente:

En forma tradicional, se ha definido al adulto conforme a su edad; es decir, a toda persona que oscila entre dieciocho y setenta años. Así, la adultez se extiende desde los veinte años y se clasifica en tres tipos: la edad adulta temprana (de veinte a cuarenta años, la edad adulta intermedia (de cuarenta a sesenta y cinco años) y la edad adulta tardía (después de los sesenta y cinco años de edad) ; no obstante, desde una concepción biopsicológica, el grupo andragógico de Nottinghan (1983) citado en Cazau, 2003, Definiciones de adulto, párr. 4), define al adulto como: "un ser en desarrollo continuo y el cual, heredero de su infancia, salido de la adolescencia y en camino hacia la vejez, continúa buscando la plenitud de sus facultades como ser humano".

Como complemento de lo anterior, la definición de adulto está vinculada al desarrollo biológico, psicológico y social de este, para responder a las interrogantes: ¿cuáles son las diferencias entre un niño y un adulto? y, por ende discernir, ¿cómo aprende un adulto? Respecto de la primera, cabe indicar que las principales características de un adulto son la capacidad de procrear, de asumir responsabilidades sociales y de decidir sobre sí mismo plenamente. Por tanto, el adulto es autónomo en el aspecto económico y social, capaz de autodirigirse, ejerce una función activa como ciudadano, forma parte de la fuerza laboral y, se inclina por decidir con base en la inteligencia más que el instinto. La praxis andragógica en los adultos de edad avanzada (como se citó en Cazau, 2003).

Respecto de la segunda, el adulto se distingue del niño por su forma de pensar y por la naturaleza de las acciones que realiza. De acuerdo con lo anterior, el pensamiento formal-operacional es activado cuando se tiene aptitud y motivación hacia el contenido por aprender. Este pensamiento inicia con una base dialéctica, que conlleva a un proceso de evaluación y reevaluación; esto es, operaciones mentales que conforman el pensamiento maduro. Durante este proceso, el pensamiento 
abstracto se conjuga con la experiencia concreta, para descubrir problemas y respuestas en lugar de la simple determinación de respuestas. Por ello, la Andragogía ha abrigado al pensamiento dialéctico por considerarlo más eficaz e impulsar su desarrollo.

En cuanto a los postulados que rigen la Andragogía se mencionan los siguientes:

1. El autoconcepto del adulto: el adulto es una persona autodirigida.

2. La acumulación de experiencias previas: estas experiencias se convierten en un recurso importante en el entorno educativo.

3. La disposición del aprendizaje por parte del adulto: este se motiva a aprender si puede relacionar lo aprendido con sus funciones sociales.

4. La aplicación del conocimiento: el adulto desea su aplicación inmediata mediante la resolución de problemas (Álvarez, 1977; Instituto Nacional para la Educación de Adultos [INEA], 2007).

Y, por su parte, los siete elementos son:

1. Establecer un ambiente adecuado: se debe propiciar un ambiente cálido, de diálogo y de respeto mutuo en el cual los participantes interactúen sin temor.

2. Planeamiento de la lección: el facilitador de la sesión debe planificar, concienzudamente, el tema y la metodología por usarse y explicar cuál es el propósito de cada uno de los procedimientos (técnicas) para llegar al descubrimiento del nuevo conocimiento.

3. Diagnóstico de las necesidades de estudio: se debe construir un modelo basado en competencias (conocimientos, habilidades y actitudes) que intervienen en el proceso educativo del aprendiente, con el fin de ayudarlo.

4. Establecer objetivos: consiste en transformar las necesidades detectadas en el elemento anterior, para convertirlas en objetivos significativos y medibles.

5. Elaborar un plan de estudios: es elaborar un programa que contenga objetivos, recursos y estrategias para alcanzar los objetivos.

6. Realizar actividades de estudio: investigación individual, debates, conferencias, diálogos, entrevistas, panel, lecturas, juego de roles, análisis de casos, asesorías, etc.

7. Evaluar los resultados del estudio: se deben desarrollar instrumentos eficientes para evaluar los resultados del proceso andragógico (Instituto Nacional para la Educación de Adultos [INEA], 2007).

Entonces, retomando el hilo conductor sobre la historia de la andragogía, se parte de la necesidad de educar al adulto para transformarlo en proletario y, de las premisas ya mencionadas, se torna inminente la institucionalización de la Andragogía dentro del sistema de educación general en Europa, Norteamérica y Latinoamérica, en las décadas de los sesenta y ochenta.

El punto de partida lo constituye la Segunda Conferencia Mundial de Educación de Adultos, propuesta por la UNESCO y llevada a cabo en Montreal en 1960. En esta oportunidad, los resultados se concretan en la formulación de objetivos comunes para implementar la Educación de Adultos, según las peculiaridades de cada país y la toma de conciencia por parte de los docentes, para teorizar sobre Andragogía y justificar su empleo en el proceso de aprendizaje de los adultos. Asimismo, la UNESCO crea dos comités internacionales (Comité Internacional de Expertos en 
Alfabetización y el Comité Internacional para el Avance de la Educación de Adultos), que se dedican a plantear políticas y teorías, que ayudan al desarrollo de la Andragogía.

En virtud de lo anterior, muchas instituciones universitarias, asociaciones profesionales de Educación de Adultos, organizaciones privadas, religiosas, culturales y políticas que requieren la Educación de Adultos dentro de su desempeño apoyan, contundentemente, el trabajo generado por la UNESCO.

Del mismo modo, la Segunda Conferencia Mundial de Educación Universitaria para Adultos en 1970, celebrada también en Montreal, gracias al auspicio de la UNESCO y de la Universidad de Montreal, profundiza sobre la pertinencia científica de la Andragogía, al discutir la ponencia del andragogo venezolano Félix Adam intitulada Andragogía: Ciencia de la Educación de Adulto, lo cual desemboca en el estudio de esta incipiente disciplina. Al mismo tiempo, esta conferencia acoge, con beneplácito, la labor iniciada por algunas universidades europeas y les marca la pauta, a fin de que estas sienten las bases institucionales, para estructurar currículos destinados a la enseñanza de la Andragogía.

En el continente europeo, Yugoslavia, Servia y Holanda son los pioneros en institucionalizar la Andragogía. Así, en Yugoslavia la educación profesional continua y la especialización en Andragogía es importante desde cursos de grado hasta doctorales. En Holanda, desde la década de los años setenta se establecen cátedras de Andragogía inclusive hasta el nivel de Doctorado. En menor grado, Polonia, Alemania, Inglaterra y Francia también la han establecido como materia impartida en algunas universidades.

En Norteamérica, Canadá se destaca por la iniciativa de establecer la Andragogía dentro de la Educación Superior. Desde 1970, se funda el Departamento de Andragogía en Montreal, que imparte Maestría y Doctorado en dicha disciplina. Luego, Estados Unidos, mediante el propulsor Malcolm Knowles, aporta al pensamiento andragógico.

En cuanto a Latinoamérica, la Educación de Adultos está relacionada con los programas educativos de alfabetización, los cuales se estiman como una estrategia de compensación ante la deficiencia del sistema regular. No obstante, en 1970 la Andragogía cobra una magnitud a partir de la creación de la Federación Interamericana de Educación de Adultos (FIDEA), organismo que se encargará de problematizar la teoría y la práctica de la Educación de Adultos y, en especial, de la Andragogía.

Por consiguiente, surge la necesidad de institucionalizar la Andragogía en las siguientes universidades: Universidades de San Marco y Garcilazo de la Vega en Perú (1976), Universidad Pedro Enrique Ureña en República Dominicana (1972-1973) y la Universidad Simón Rodríguez en Venezuela (1975). Luego, las actividades del FIDEA y la colaboración de la Universidad Simón Rodríguez impulsan, notablemente, los principios e innovaciones inherentes a la praxis de la Andragogía.

En 1981, en Colombia, nace el Instituto Internacional de Andragogía (INSTIA), el cual expande los principios y prácticas andragógicas y los enriquece mediante la investigación socioeducativa, el ofrecimiento de programas de capacitación y de postgrado y el desarrollo de acciones de cooperación con instituciones vinculadas a la práctica de esta disciplina (Álvarez, 1977).

Por otro lado, el andragogo Adam (1984) sostiene que la enseñanza universitaria latinoamericana debe renovarse; esto es, abandonar la pedagogía y acogerse al acto andragógico, ya que el estudiantado es adulto y como tal, tiene un perfil psicosocial definido y una forma de aprender estudiada por esta última ciencia, las cuales ya fueron explicitadas en este artículo. 
Lo anterior, implica, esencialmente, una adecuación de los aprendizajes y una horizontalización en la relación estudiante-profesor. Al respecto expresa Adam (1984) en la siguiente cita:

(...) el docente universitario tiene el dominio cognoscitivo como objetivo (transmitir conocimientos) mas no es el metodológico de ofrecer su experiencia profesional a quienes considera sus "alumnos", dentro de la concepción pedagógica, ya que la educación universitaria sigue regida en su organización y administración educativa por los principios de dependencia (...). (p. 4)

Para ello, las universidades deben asumir el compromiso de formar a su personal académico y administrativo en la tecnología andragógica. Pero, ¿de qué forma? Se debe capacitar a los docentes universitarios en ejercicio y disponer como requisito para los futuros la Licenciatura y la Maestría en Andragogía, para conocer la problemática de la Educación de adultos en cuanto a planeamiento y a administración de programas y, finalmente, a través del Doctorado incentivar la investigación en esta área (Adam, 1984).

\section{El acto andragógico como propulsor de las competencias cognitivas y metacognitivas}

La psicóloga cognitiva Sanz (2010) explica que la tendencia actual, en Europa, para organizar el currículo universitario son las competencias. Estas se entienden como las habilidades (capacidad específica), los conocimientos, las actitudes y las experiencias que utiliza una persona, para resolver, de forma adecuada, una tarea en un contexto definido. Así pues, las competencias se vinculan con elementos mentales, culturales, actitudinales y conductuales, que resaltan la aplicación de conocimientos y de procedimientos en el saber hacer.

Asimismo, ella comenta que las competencias denominadas genéricas son deseables de desarrollarse en el currículo universitario, independientemente, del área de conocimiento por estudiar. Dentro de ellas, se encuentran las competencias cognitivas, tales como el pensamiento comprensivo, el pensamiento crítico, el pensamiento creativo, la toma de decisiones y la solución de problemas, que permiten las siguientes operaciones mentales: el análisis, la síntesis, las habilidades críticas, la valoración de la información y la generación de ideas. Todas ellas serán descritas de seguido.

La primera se ocupa de la comprensión y de la expresión de significado en diversas experiencias, situaciones, datos, creencias, reglas, procedimientos o criterios de los ámbitos físico, social, cultural y profesional, sobre los que el estudiante debe reflexionar y juzgar, para resolver problemas de forma eficiente; luego, la segunda, se refiere al acto de evaluar la información, a fin de inferir la autenticidad del razonamiento que esta genera, para después emitir juicios de valor de aceptación o de rechazo. Para ello, es imprescindible investigar la solidez de las fuentes de información, la interpretación de las causas, con el objeto de propiciar siempre un mejoramiento continuo de las experiencias de aprendizaje; predecir efectos de eventos actuales, para evitar la repetición de desaciertos cometidos en el pasado; razonar analógicamente, para emplear la experiencia y conocimiento en la resolución de nuevos problemas y también pensar deductivamente, lo cual permitirá acomodar las ideas y obtener conclusiones sobre los distintos argumentos. 
La tercera se define como la capacidad de producir ideas originales y combinarlas, de manera que se establezcan nuevas relaciones entre ellas, a través de su visualización y de su comparación, para generar otras que se echarán a andar en futuros proyectos. La cuarta consiste en dictaminar sobre un hecho, a partir del análisis de los factores que lo influyen, de las opciones disponibles y de las posibles consecuencias, para luego resolver el problema, a fin de decidir con base en la opción que asegure el logro de la meta. La quinta es la resolución de un problema, proceso que inicia con el análisis de la situación actual de él, para planificar la estrategia que conllevará a su desenlace. Cabe destacar que esta competencia es la más compleja de todas, ya que se requiere del uso de todas las anteriores.

También, se encuentran las competencias metacognitivas: la autorregulación y la transferencia. Por un lado, la autorregulación constituye un proceso mediante el cual el discente organiza, examina y juzga su experiencia de aprendizaje y su conducta dentro de esta, para planificar y ejecutar las acciones que lo llevarán al éxito; así como evaluar sus logros, para implementar mejoras en futuros aprendizajes. Por otro lado, la transferencia es la capacidad de trasladar lo aprendido de una experiencia de aprendizaje a otras nuevas, sean de la misma área de conocimiento o no, lo cual se considera la prueba de que este se ha vivenciado (Sanz, 2010).

Ahora bien, ¿cómo contribuye el acto andragógico al desarrollo de estas? Al hacer un recuento de los antecedentes de la Andragogía, pero, especialmente, de los principios andragógicos, del proceso de pensamiento del adulto y de mi experiencia laboral como profesora de español como segunda lengua en Cuerpo de Paz, Costa $\mathrm{Rica}^{3}$, se observa que el acto andragógico reproduce la mayéutica socrática, a través de la teoría de aprendizaje experiencial, estructurada en el Ciclo de aprendizaje experiencial de David Kolb, que se presenta en la siguiente figura:

Figura 1. Ciclo del aprendizaje experiencial de (Kolb citado en Gómez, s. f.)

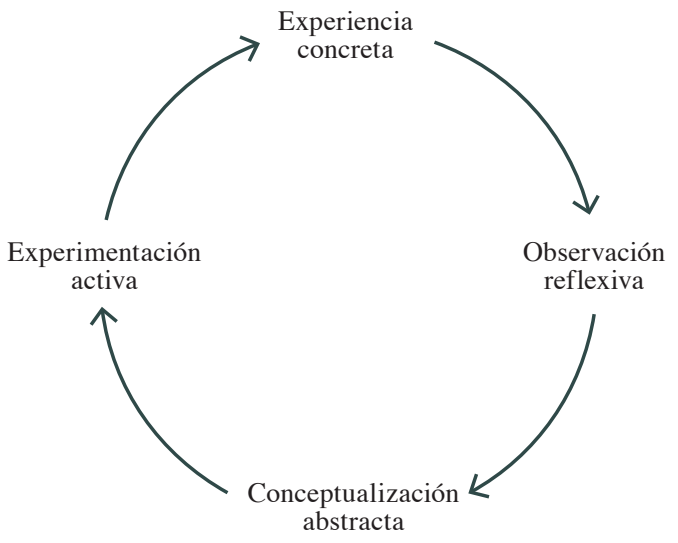

Y que ambas fases (mayéutica y ciclo de aprendizaje experiencial) organizan la consecución de los objetivos de aprendizaje mediante las competencias cognitivas y metacognitivas ya citadas. A continuación, se explican el Ciclo de aprendizaje experiencial junto con las competencias que

Cuerpo de Paz es una agencia federal del Gobierno de los Estados Unidos, que promueve el trabajo voluntario, en países tercermundistas. Su metodología de trabajo está basada en la teoría del aprendizaje por experiencia de Kolb (1984) citado en Gómez (s. f.), que se representa en el Ciclo de aprendizaje experiencial. 
se desarrollan en cada etapa, para después entender su comparación con la mayéutica. Así pues, en la primera etapa de este ciclo (experiencia concreta), se genera conocimiento a partir de una experiencia particular, entendida como el hacer algo, lo cual constituye la interacción entre el ser humano y su entorno, o desde la perspectiva de Sócrates, salirse de sí mismo para concientizarse y controlar el mundo en donde habita, lo que redundará en su autoconocimiento.

De seguido, en la segunda etapa (observación reflexiva) se razona sobre tal experiencia, al establecer una relación entre esta vivencia y los resultados obtenidos. En este punto, el alumno utiliza la síntesis; esto es, la descripción de la experiencia concreta, para responder a las preguntas del facilitador: ¿Qué pasó?, ¿Qué hicimos?, que, de seguido, despliegan el análisis; es decir, la comprensión de la experiencia y su significado dentro de su entorno inmediato. Ambas son operaciones cognitivas del pensamiento comprensivo.

Luego, en la tercera etapa (conceptualización abstracta), mediante ese análisis se cosechan generalizaciones o conclusiones, derivadas de la evaluación de la experiencia concreta. Esta actividad se logra por medio de las mencionadas habilidades críticas (investigación de fiabilidad de fuentes, interpretación de las causas, predicción de efectos, razonamiento analógico y deductivo), con el objeto de realizar inferencias que se aplicarán en la próxima etapa. Todas ellas son habilidades del pensamiento crítico. Para ello, el alumno responde a las preguntas del guía: ¿Y eso significa? ¿Qué le sugiere este tema en general? ¿Le recuerda esto algo? ¿Cómo relaciona esto a otras experiencias?

En la cuarta etapa (experimentación activa), se procede a practicar tales conclusiones en la vida cotidiana y se efectúa un plan de acción aplicable en el futuro, para afrontar circunstancias similares. Aquí, el alumno dispone de la generación de nuevas ideas, la toma de decisiones, la solución de problemas, la autorregulación y la transferencia, habilidades que corresponden al pensamiento creativo y a competencias metacognitivas, en orden respectivo y que garantizan el éxito del aprendizaje experiencial. (Gómez, s. f.) Para tal efecto, este debe responder a las preguntas ¿Cómo se relaciona lo aprendido con experiencias previas? ¿Cómo se aplica lo aprendido en la vida personal, laboral? ¿Cómo relacionará lo aprendido con eventuales situaciones futuras? ¿Cuáles son las consecuencias de hacer esta actividad? A través de estas preguntas el alumno sistematiza y reevalúa el aprendizaje y su conducta; es decir, utiliza la autorregulación, para transferirlo a circunstancias futuras y así forjar el camino hacia la perfectibilidad, que es la esencia de la educación.

Entonces, si se comparan las fases de la mayéutica con el aprendizaje por experiencia, resulta evidente que ambos parten de un problema o de una acción, que implican un enigma por descubrir (experiencia concreta), que se resolverá mediante la dialéctica entre el facilitador y el estudiante, lo cual estimula el cuestionamiento de los hechos, no solo para garantizar la veracidad de las ideas preconcebidas, sino también para suscitar otras nuevas y enriquecidas sobre dicho problema (observación reflexiva). Esta dialéctica, a su vez, genera un debate por medio de razonamientos, que establecen conceptos generales o conclusiones y, así, facilitador y estudiante evalúan el conocimiento presente, pues este debate permite que el estudiante, con la guía del facilitador, desarrolle conceptos remozados, a partir de los anteriores (conceptualización abstracta).

Por último, el papel del facilitador ha terminado, pues ya le ha ayudado a "parir" al estudiante los innovadores conceptos, que este utilizará en otras circunstancias, con lo cual se reforzará su perfectibilidad, fin último de la educación, a través de su autoconocimiento, o en palabras socráticas, conócete a ti mismo, entendido aquí como su forma de razonar y, por extensión, su forma de aprender. 
Así las cosas, es deseable que la educación superior, en otras latitudes, y específicamente, en Costa Rica, implemente el uso de la disciplina andragógica, con el objetivo de respetar la condición del adulto en el entorno educativo y formar futuros ciudadanos y profesionales pensantes; por ello, autónomos en el plano personal y profesional, inclinados a buscar el conocimiento mediante el pensamiento convergente y divergente, pues como lo manifestó Platón en La república: "No se puede obligar a nadie a aprender, ni podemos abrir las cabezas para meter los conocimientos en ellas. Solo se puede mostrar el camino, para que cada cual piense por sí mismo" (Jackson, 2004, párr. 3).

\section{Conclusiones}

La Andragogía como disciplina de la Educación es relativamente nueva y desconocida, ya que a lo largo de su trayectoria no ha sido aceptada por pedagogos, quienes argumentan que la Pedagogía está organizada para educar a todas las personas sin distingo de edad; por ello, discrepan de la fundamentación teórica de la Andragogía, a pesar de que algunos de sus lineamientos ya fueron establecidos desde la antigüedad clásica.

En contraposición, la Andragogía sí considera que se debe educar a las personas según las características propias de la edad. Así, se basa en el perfil biológico, psicológico y social de su destinatario: el adulto. Esta educación se concreta en el acto andragógico, cuyos principios orientan la función activa del estudiante a través de la revelación del conocimiento, a partir del análisis.

Finalmente, se verifica que el acto andragógico permite el desarrollo de las competencias cognitivas y metacognitivas, las cuales deberían enseñarse, paralelamente, al conocimiento académico de cada disciplina impartida en las universidades, a fin de brindar una educación integral al estudiantado.

\section{Referencias}

Adam, F. (1984). Universidad y educación de adultos. En Siete visiones de la educación de adultos (pp. 86-93). Pátzcuaro, México: CREFAL. Recuperado de http://www.crefal.edu.mx/ crefal2012/images/stories/publicaciones/retablos papel/retablo papel1.pdf

Álvarez, A. (1977). Análisis crítico de la Andragogía en base a las ideas de Knowles, Adam y Savicevic. (Tesis doctoral). Universidad Nacional Experimental Simón Rodríguez. Caracas, Venezuela. Recuperado de http://postgrado.una.edu.ve/andragogia/paginas/alvarez1977.pdf

Banchio, L. (10 de febrero del 2004). La educación según Platón. [weblog post]. Recuperado de http://www.luventicus.org/articulos/04D001/index.html

Cazau, P. (11 de agosto del 2003). ¿Qué es la Andragogía? [web logpost]. Recuperado de http:// articulosdeyca.blogspot.com/2009/03/que-es-andragogia.html 
Fingermann, H. (9 de marzo del 2011). Sócrates y la Educación [weblog post]. Recuperado de http://educacion.laguia2000.com/general/socrates-y-la-educacion

Gómez, J. (s. f.). El aprendizaje experiencial. [Cód. 693, curso Capacitación y desarrollo en las organizaciones, Facultad de Psicología, Universidad de Buenos Aires]. Buenos Aires, Argentina. Recuperado de http://www.ecominga.uqam.ca/ECOMINGA 2011/PDF/ BIBLIOGRAPHIE/GUIDE LECTURE 5/1/3.Gomez Pawelek.pdf

Hernández, P. (s. f.). El pensamiento educativo de Aristóteles. Recuperado de http://scarball. awardspace.com/documentos/trabajos-de-filosofia/Aristoteles.pdf

Instituto Nacional para la Educación de Adultos (INEA). (2007). Andragogía (Lectura 1, Año 9). México, D. F. Recuperado de http://tecnoeduka.110mb.com/documentos/teoria\%20 aprendizaje/Andragogia\%202.pdf

Jackson, R. (11 de noviembre de 2004). Redacción: Educación en Platón [weblog post] Recuperado de http://dolphin.blogia.com/2004/111102-redaccion-educacion-en-platon.php

Lépiz, C. (2011). Perspectiva de la docencia universitaria [Presentación Power point]. Heredia: Universidad Nacional.

Mijangos, R. (s. f.). Juan Federico Herbart: Propuesta pedagógica. Recuperado de http://scarball. awardspace.com/documentos/trabajos-de-filosofia/Herbart.pdf

Pascual-CastroViejo, I. (1996). Plasticidad cerebral. Rev Neurol, 24(135), 1361-1366. Recuperado de http://www.psicomag.com/biblioteca/1996/Plasticidad\%20Cerebral.pdf

Rivera, A. (1998). Arqueología del lenguaje en el proceso evolutivo del género homo. espacio, tiempo y forma, Serie I, Prehistoria y Arqueología, 11, 13-43. Recuperado de http:// e-spacio.uned.es/fez/eserv.php?pid=bibliuned:ETFSerie1-E9059635-79A4-9C64-E22AB9146E7D8EDC\&dsID=PDF

Sánchez, P. A. (1989). Hacia una estrategia peda-andragógica que incremente la participación del estudiante en el aprendizaje de la Matemática (un enfoque antinómico). (Tesis de Maestría). Universidad Nacional Experimental de los Llanos Occidentes Ezequiel Zamora (UNELLEZ), Barinas, Venezuela. Recuperado de http://servidor-opsu.tach.ula.ve/ascen_acro/sanc_al/cont/ presentacion.pdf

Sanz, M. L. (2010). Competencias cognitivas en Educación Superior. Madrid: Narcea.

Tarrio, J. A. (s. f.). La educabilidad. Recuperado de http://webs.uvigo.es/jtarrio/OBRA LITERARIA/LA EDUCABILIDAD.pdf 